\title{
Algunas reflexiones sobre bibliotecas históricas 0 patrimoniales: nuevo paradigma entre los centros y servicios de información
}

\author{
Manuel José Pedraza Gracia
}

Artículo recibido: 20 de mayo de 2013. Artículo aceptado: 27 de febrero de 2014.

\section{Resumen}

Se analizan las características específicas que poseen las bibliotecas históricas o patrimoniales que permiten individualizarlas entre todos los tipos de centros bibliotecarios existentes. Para ello, en primer lugar, se examinan las características del libro antiguo como documento y como objeto; en segundo lugar, se estudia el libro antiguo como conglomerado de informaciones útiles para la investigación; en tercer lugar, se considera la relación existente entre las unidades documentales y las bibliotecas patrimoniales; en cuarto lugar, se profundiza en la vertiente patrimonial de la biblioteca y sus implicaciones. Finalmente se examinan las funciones de este tipo de bibliotecas y la manera en la que

* Universidad de Zaragoza, España. pedraza@unizar.es

INVESTIGACIÓN BIBLIOTECOLÓGICA, Vol. 28, Núm.64, septiembre/diciembre, 2014 México, ISSN: 0187-358X. pp. 33-50 


\begin{abstract}
éstas influyen en las actividades y actuaciones que se realizan en ellas.
\end{abstract}

Palabras clave: Patrimonio bibliográfico; Bibliotecas patrimoniales; Libro antiguo; Fondos históricos.

\begin{abstract}
Reflections on historical and heritage libraries: The emerging paradigm among information service centers Manuel-José Pedraza-Gracia

The paper analyzes the special features of heritage libraries that make them distinct from other library centers. Rare books are examined as documents and heritage objects, as well as reservoirs of useful information for researchers. The linkage between document archives and heritage libraries is also studied. Finally, the main functions of heritage libraries are described in terms of their impact on institutional activities and agenda.
\end{abstract}

Keywords: Bibliographic heritage; Heritage libraries; Rare books; Special collections.

\title{
INTRODUCCIÓN
}

T os libros antiguos, las bibliotecas que poseen fondos antiguos y las biLbliotecas que se conservan como en tiempos pretéritos ${ }^{1}$ causan cierto sobrecogimiento entre los que las visitan. Esa impresión, la de retroceder en el tiempo y vivir el pasado, es idéntica a la que se siente cuando se observa un

1 Hay una casuística muy diversa en las bibliotecas que poseen fondos antiguos: las hay que poseen solamente libros antiguos o las que poseen además libros modernos, y entre las primeras las hay que mantienen la biblioteca de la institución como estaba en el pasado (sin nuevas incorporaciones) o las hay que siguen adquiriendo nuevas unidades y bibliotecas completas; y, entre las segundas, las que mantienen el fondo histórico de la entidad sin más adquisiciones y las que crean una colección de libros antiguos para acompañar a los fondos modernos. Pero no existe un consenso sobre la denominación que se puede dar a este tipo de centros: biblioteca histórica, biblioteca-museo, biblioteca patrimonial, biblioteca de investigación; ni lo hay cuando se trata de una sección de la biblioteca: colecciones especiales, fondo antiguo, fondo patrimonial, tesoro, reserva, entre otros muchos. Un análisis de todos estos conceptos y sus implicaciones se está preparando en la actualidad. 
objeto antiguo o se entra en un edificio histórico. Pero las sensaciones son subjetivas por lo que pueden no ser las mismas en dos individuos, ni poseen la misma intensidad cuando alguien se encuentra ante bienes que pertenecen a su propia cultura que cuando pertenecen a culturas ajenas. En el punto extremo de esta afirmación existen numerosísimos ejemplos de individuos y de pueblos que despreciaron las culturas de otros cuando alcanzaron posiciones predominantes sobre éstos, dando como resultado, en la mayoría de las ocasiones, pérdidas irreparables para las culturas afectadas y para la humanidad en general. ${ }^{2}$

Parece evidente, a la luz de la experiencia, que los libros y fondos antiguos poseen algo más que la mera información textual y los contenidos formales (autor, título...) que caracterizan a los documentos modernos. ${ }^{3}$ Ésta es una característica propia de estos fondos cuya repercusión en la biblioteca y en su proceder ha de ser analizada porque, aunque se ha iniciado el argumento tratando de libros y bibliotecas, las afirmaciones que se acaban de enunciar no suelen ser consideradas dentro del conjunto de reflexiones que la bibliotecología realiza sobre su objeto de estudio, y tampoco ha sido contemplada la incidencia que este cúmulo de impresiones y sensaciones tiene en la forma de actuar y de gestionar la biblioteca por parte de la biblioteconomía. ${ }^{4}$ Este vacío implica obviar las potencialidades de primer orden que atesoran las bibliotecas que poseen libros y fondos históricos ${ }^{5}$ con respecto a las sociedades que sirven, las cuales adoptan rigurosas medidas de carácter normativo cuando son conscientes de ese potencial cultural.

Este trabajo pretende ofrecer una serie de reflexiones sobre estos aspectos con objeto de llegar a apreciar si las bibliotecas con fondos antiguos poseen un conjunto de características que permitan considerarlas como una clase diferente de las tipologías de los centros informativos.

2 Cf. entre otras obras sobre esta temática las de Fernando Báez, Historia universal de la destrucción de libros, Barcelona: Destino, 2004, y Nueva historia universal de la destrucción de libros, Barcelona: Seix Barral, 2011.

3 Vid. dos de las obras clásicas que han sentado las bases de la bibliografía material anglosajona y que justifican estas apreciaciones: Ronald B. McKerrow, Introducción a la bibliografía material, Madrid: Arco/Libros, 1998, trad. de la edición de 1994, aunque su edición original es de Oxford, Clarendon Press, 1927; y Philip Gaskell, Nueva introducción a la bibliografía material, Gijón: Trea, 1998, trad. de la edición original de Oxford, Oxford University Press, 1972.

4 Una excepción a esta aseveración es el manual recopilado por José Antonio Magán Wals, Temas de biblioteconomía universitaria y general, Madrid: Universidad Complutense, 2002, que contiene un interesante capítulo firmado por Manuel Sánchez Mariana al respecto de los fondos antiguos.

5 Tal es la afirmación anterior que no existe una denominación consensuada para las bibliotecas con fondos antiguos. Cf. Manuel Carrión Gútiez, "Tres lecciones sobre la Biblioteca Nacional de España”, en Ramón Rodríguez Álvarez y Moisés Llordén Miñambres, El libro antiguo en las bibliotecas españolas, Oviedo: Universidad de Oviedo/Servicio de Publicaciones, 1998, pp. 11-14. 


\section{EL LIBRO ANTIGUO COMO PRODUCTO INDIVIDUALIZADO}

El proceso de elaboración de los libros durante el periodo manual y mecánico de la imprenta mantiene las características propias generales de este tipo de producción. Se pueden ver algunos casos:

a) La detección de errores, los accidentes y los imprevistos debían resolverse sobre la marcha dando lugar con cada solución a conjuntos de productos que se diferencian con claridad de otros dentro de la misma serie.

b) La posibilidad de recuperar siquiera parte de la inversión efectuando pequeñas modificaciones o acciones sobre productos de difícil salida que han quedado en los almacenes durante un tiempo.

c) La elaboración de nuevas series de un producto ya distribuido debido al éxito obtenido con el mismo.

En los talleres en los que se elaboraban libros impresos se seguían actuaciones similares que daban lugar a ediciones, emisiones y estados cuya identificación presenta en la actualidad una complejidad notable, ya que exigen en la mayoría de los casos la comparación de ejemplares, lo que no siempre es posible. $^{6}$

El sistema de producción manual del libro impedía que las formas de imprenta se mantuviesen montadas durante más tiempo del necesario para imprimir la tirada del pliego correspondiente y, en consecuencia, que la reelaboración de una forma de imprenta llegase a producir otra exactamente idéntica a una preparada previamente. Por esta causa en el periodo manual de la imprenta era prácticamente imposible hablar de reimpresión, que solamente tenía sentido en la tirada de productos de carácter efímero de uso continuado, para cuya elaboración podían mantenerse montadas formas confeccionadas con tipos en desuso reservados en exclusividad para este fin y que servirían para mantener en funcionamiento las prensas en los momentos en los que se hubiese de interrumpir el trabajo con el pliego en curso de impresión. Una edición no puede ser, por consiguiente, idéntica a otra, a diferencia de lo que ocurre con las ediciones de libros contemporáneos. La mención de la edición o edición en el propio documento ni es preceptiva ni frecuente en la época, presentándose, por añadidura, el problema de la identificación de ediciones falsificadas y contrahechas como consecuencia de la normativa.

6 Cf. la primera aportación en castellano sobre este aspecto del maestro Jaime Moll, "Problemas bibliográficos del libro del Siglo de Oro”, en Boletín de la Real Academia Española, 59, 216, 1979, pp. 49-107. 
Existen además diversas actuaciones, premeditadas o no, que se efectúan durante o después de la tirada y que producen emisiones y estados dentro de las ediciones, lo cual da origen a conjuntos de ejemplares con características diferentes dentro de la misma edición. ${ }^{7}$

El taller de imprenta es durante todo el periodo solamente el lugar en el que se imprimen los libros. Éstos, posteriormente, eran comercializados o llevados en rama a la librería, que era el comercio del artesano que hacía los libros. En la librería se confeccionaba el libro dotándolo de encuadernación. Un porcentaje de ellos, a juicio del librero, se encuadernaba en pergamino flexible, encuadernación tan frecuente y característica del libro hispano, pero otro porcentaje se reservaba para que el librero los encuadernara siguiendo las indicaciones del comprador sobre los materiales y las decoraciones e incorporaba los signos de propiedad que le solicitaban en los lomos o en las cubiertas.

Por consiguiente, entre los ejemplares supuestamente idénticos pertenecientes a una misma edición pueden hallarse variedades y disimilitudes que los individualizan, a lo que hay que añadir que, si no se encuadernan con la tradicional encuadernación de pergamino flexible, poseerán con bastante seguridad características bibliopégicas exclusivas.

El libro antiguo es también un producto histórico en cuanto los acontecimientos inciden en él. Seguramente algunos ejemplares idénticos, no todos como se ha visto, llegaban a manos de diferentes poseedores, y a partir de ese momento cada ejemplar seguía un íter que tenía como resultado la incidencia sobre él de factores de muy diversa índole y conformaba un ejemplar con características propias distintas de las de los otros ejemplares de la edición que poseen, a su vez, sus características únicas.

Hacer una exhaustiva enumeración de todos esos factores que inciden a lo largo de periodos de tiempo, en ocasiones muy amplios, en singularizar el ejemplar sería el objeto de otro trabajo. El principal, sin duda, es la propia destrucción que ha dado como resultado numerosas ediciones de las que apenas se conservan ejemplares u otras muchas que se han perdido para siempre. La desaparición de los semejantes dota de una característica especial e intangible a los que permanecen, la de la rareza. El uso y el vandalismo es causa también de la existencia de ejemplares mutilados, en ocasiones los únicos restos de una edición. Con respecto a otros factores de diversificación, bastará con citar algunos que inciden de forma muy diversa sobre el ejemplar. Los hay que afectan a los contenidos textuales, como el expurgo, las incorporaciones de elementos manuscritos o de marginalia; los hay que 
inciden sobre el aspecto físico, como el ataque de insectos bibliófagos, la humedad o los hongos, la encuadernación o, incluso, las sucesivas encuadernaciones; los hay que atañen a la estética, como la perfecta conservación, la decoración de los cortes o el coloreado de las ilustraciones, etcétera. Además, los diversos poseedores incorporan marcas de propiedad, anotaciones manuscritas, color a los grabados o decoración ex novo, conforman volúmenes factícios o intercalan entre las hojas impresas otras en blanco para efectuar anotaciones, entre otros elementos.

Por lo tanto, se puede mantener que es prácticamente imposible encontrar dos ejemplares idénticos de un libro antiguo y que esto es mucho más difícil cuanto más antigua sea la edición porque con el transcurso del tiempo ha habido más posibilidades de intervención de la naturaleza y del ser humano. La consecuencia bibliotecológica principal de estas precisiones es que uno de los usos de los fondos bibliográficos antiguos es la comparación entre ejemplares con objeto de hallar similitudes y diferencias entre ellos.

Estas aseveraciones conducen a una primera afirmación: los ejemplares antiguos componentes de una biblioteca o un fondo bibliográfico son, tanto por su proceso de fabricación como por el trascurso del tiempo, ejemplares con características propias que los hacen únicos y, en consecuencia, imposibles de reponer en caso de pérdida. De la misma manera, un ejemplar extraído o sustraído de una determinada biblioteca posee características propias que lo hacen perfectamente reconocible como perteneciente a esa biblioteca a pesar del trascurso del tiempo, lo que facilita la reconstrucción de las bibliotecas del pasado cuando no han sido destruidos sus elementos componentes.

\section{EL LIBRO ANTIGUO COMO CONGLOMERADO DE INFORMACIONES ÚTILES PARA LA INVESTIGACIÓN}

Como se ha podido percibir, cuando se trata de un libro antiguo lo que el usuario contemporáneo encuentra es algo más que un texto soportado en el tipo documental denominado codex. De hecho, no es frecuente que lo que se busque, sin más, sea el texto.

Por lo que respecta a los textos literarios contenidos en los libros antiguos, generalmente han sido editados con posterioridad y completados con los análisis de investigadores y editores literarios que dotan a los textos de un valor añadido que jamás poseyeron los ejemplares de su edición princeps o en los de sus primerísimas ediciones. En cuanto a los textos científicos, los progresos contenidos en ellos suelen ser superados por investigaciones posteriores que toman como fundamento los conocimientos expuestos en 
las ediciones de obras precedentes. Solamente aquellos trabajos que pretenden justificar o revisar dichas investigaciones anteriores son requeridos para aportar nuevos y propios valores añadidos. Sólo aquellos textos poco conocidos que aportan informaciones escasamente difundidas se utilizan de la misma manera que el libro moderno: con objeto de acceder a su texto. Por consiguiente, no pueden considerarse estas aproximaciones a los textos de los libros antiguos las que principalmente precisan de su utilización. ${ }^{8}$ Se ha de tener en cuenta que el uso de los libros antiguos exige un conocimiento de sus características y cierta experiencia en su manejo, lo que no los hace fáciles para usuarios no preparados. Para ello se emplean medios que protegen el documento de su utilización inadecuada.

Entonces, ¿qué se busca en el libro antiguo? Las respuestas son variadas. La más evidente, y también más frecuente, es que el libro antiguo es fuente principal para el estudio de la obra y del libro; es éste un campo de investigación más amplio de lo que pueda suponerse en principio. Esta afirmación se sustenta en las múltiples posibilidades de análisis que posee. ${ }^{9}$

Determinadas condiciones comerciales, legales e incluso publicitarias en la evolución del libro llevaron a que los libros antiguos posean una estructura formal en la que el texto se ve envuelto por un conjunto de piezas de muy diversa índole que reciben el nombre de paratextos, en los libros actuales completamente perdidos, que proporcionan una información sobre la obra y sobre la elaboración del libro que trasciende al propio texto. El análisis de esta estructura, solamente equiparable al análisis diplomático de los documentos manuscritos, es de tal trascendencia que permite obtener información sobre los autores y sus relaciones, sobre la obra en lo referente a determinados aspectos de su génesis y, de forma similar, sobre la edición, de tal suerte que pueden ayudar a determinar los datos imprescindibles para su identificación. Bien es cierto que esta estructura es fruto de una evolución que se "perfecciona" con

8 Carrión define perfectamente la condición de libro antiguo con las siguientes palabras: "Asciende el libro a esta condición, cuando deja de interesar por lo que dice (trasvasado ya generalmente a nuevas y 'mejores' ediciones o incorporado a estudios nuevos que han venido a incrementar el conocimiento social) e interesa por lo que es: testimonio y objeto cultural de un tiempo [...] El interés se centra en el libro todo entero [...] no es que, en muchos casos, los libros hayan perdido su condición de 'libro instrumento' ni que se hayan reducido a la condición de 'libro objeto' (valioso por su condición de obra de arte, por su rareza o por su antigüedad), sino que han pasado a ser objetos culturales, testimonios para la historia de la comunicación, campo para la historia del libro, en esa tierra de nadie que dejan libre o a medio ocupar otras disciplinas históricas y para la bibliografía material a mitad de camino entre la historia y la crítica textual". Op. cit., p. 12.

9 Cf. sendas aproximaciones a los valores que posee el libro antiguo sólo apreciables mediante su análisis: Manuel José Pedraza Gracia, "La valoración de fondos antiguos en bibliotecas", en Ibersid: revista de sistemas de información y comunicación, 2, 2008, pp. 263-272; Julián Martín Abad, La valoración del libro: el punto de vista del bibliotecario de fondo antiguo, Documentos de trabajo U.C.M. Biblioteca Histórica, 8, 2004, pp. 1-25. 
el tiempo, por lo que cuanto más antiguo es un libro aquélla resulta ser más "imperfecta".

El libro antiguo posee, también, una estructura material conformada por el conjunto de elementos materiales que lo constituyen. Entre ellos se encuentran el papel, la tinta y los restos que dejan sobre el soporte otros materiales, como los tipos metálicos y xilográficos, que conjuntamente conforman el libro como objeto histórico; es esta faceta de investigación no muy diferente a la de cualquier otro objeto histórico. El análisis de esta estructura material y la investigación de los elementos materiales que intervienen en la confección del producto bibliográfico son fundamentales para su conocimiento y su evolución histórica y elemento de imprescindible análisis para la identificación de impresos sine notis. Las aportaciones que se realizan al ejemplar con el transcurso del tiempo son también estudiadas. Se trata, por consiguiente, de un análisis que posee una perspectiva diacrónica sobre el objeto de estudio que ha poseído una historia propia en la que él mismo ha evolucionado. No se ha de olvidar que la influencia material sobre la obra es una vertiente de la investigación científica ya muy reconocida dentro de la bibliografía.

También existe una vertiente de análisis estético y emblemático que afecta a aspectos tan sustanciales como la decoración y la ilustración. En el libro antiguo ambos se introducen, a la par que los textos, en la conformación de los pliegos, pero también como decoración o marca de propiedad en las encuadernaciones, en los exlibris, sellos, marcas de fuego y dibujos que aportan los sucesivos propietarios al ejemplar en cuestión.

Son muchos los intereses que despiertan entre los investigadores los libros antiguos y son muchas las causas que llevan al investigador a la consulta de los fondos antiguos, pero no puede considerarse que sea la simple consulta de los textos la principal de ellas. De hecho, es el estudio del propio libro en sus múltiples facetas el foco principal de estudio e investigación.

Pero esta situación es realmente reciente. Hasta hace relativamente poco tiempo los fondos antiguos se poseían y se contabilizaban, pero suponían más un problema que otra cosa: fondos poco utilizados, difíciles de comprender por el idioma (frecuentemente el latín), ciertamente trasnochados en sus contenidos y difíciles de conservar, por lo que bibliotecológicamente no eran apreciados.

\section{LA UNIDAD Y EL CONJUNTO, LA PIEZA Y LA BIBLIOTECA}

Se ha visto la trascendencia para la investigación o, mejor, para una importante casuística relacionada con la investigación, que puede tener el libro 
antiguo como unidad. Pero las unidades bibliográficas no surgen de manera espontánea, generalmente provienen de un conjunto o de una biblioteca que fue originalmente conformada bajo unas condiciones y unos condicionamientos propios de quienes las crearon y de los tiempos en los que las originaron, y que han llegado hasta la actualidad de forma más o menos íntegra. La obsolescencia de los contenidos fue relegando a un conjunto de libros a una especie de limbo del que difícilmente salían salvo por el interés de algún investigador que (re)descubría alguna joya bibliográfica entre los fondos menos solicitados por los usuarios de una biblioteca. De esta manera se constituían de forma independiente dos fondos o sub-bibliotecas: la que merecía fondos, personal, dedicación con fondos modernos y actualizados y la que podía, a lo sumo, anunciar lo longevo de la institución.

Pero esta premisa marca un nuevo objeto de investigación, el estudio de los conjuntos bibliográficos, de las colecciones, de las bibliotecas. Se ha intentado poner de manifiesto la importancia del análisis de las procedencias porque se trata de una fuente de primera importancia para el conocimiento de las personas y de las instituciones que poseen esas bibliotecas. Son muchas las cuestiones que se pueden observar cuando se analizan minuciosamente, unidad por unidad, los contenidos de una colección o biblioteca: los intereses del propietario (persona física o institución) sobre temas y materias, sobre corrientes filosóficas, políticas o religiosas; niveles y capacidad de conocimientos, idiomas que maneja o lee el propietario, relaciones personales o intelectuales con autores contemporáneos, constitución de una biblioteca profesional personal, ausencias notorias y presencias extrañas, etc. Este tipo de análisis proporciona una instantánea de la situación de la persona o de la institución que creó y poseyó la biblioteca que sería imposible estudiar con otra fuente. Las colecciones y bibliotecas suelen terminar formando parte de otras bibliotecas que las adquieren por diversos procedimientos perfectamente conocidos: compra, legado, depósito, donación o dación, entre otros. Las bibliotecas receptoras adquieren aquí una nueva responsabilidad, la de mantener el conjunto perfectamente identificado de forma que su estudio no quede truncado por una incorrecta integración de la biblioteca recuperada en la biblioteca receptora. En estos casos el objeto de estudio es múltiple: las unidades bibliográficas como piezas singulares, por una parte, y la colección como objeto plural, por otra.

Asimismo, el libro como objeto de estudio no puede ser tratado como una pieza aislada sino que más bien requiere el conocimiento del contexto, de la colección en la que estuvo integrado. Esta particularidad es inherente a los elementos que constituyen el patrimonio cultural cuyo estudio precisa, para su perfecta comprensión, el conocimiento del entorno para el que se concibieron, se seleccionaron o en el que alcanzaron su utilidad. 
Este tipo de estudios no era frecuente en las bibliotecas con fondos antiguos, frecuentemente conjugados con los modernos, salvo, quizás, en las que se podrían denominar como biblioteca-museo (como la Real Biblioteca del Monasterio de El Escorial, la Biblioteca Palafoxiana o la Herzog August Bibliothek de Wolfenbüttel), caracterizadas por haberse convertido en la instantánea de una biblioteca tipo en un momento histórico específico. Estas bibliotecas, que se mantenían paralizadas en el pasado, en momentos recientes han tomado conciencia de ser centros de información que pueden poner en valor sus fondos para la investigación. Además de estas bibliotecas-museo, surgen ex novo otras que se concentran en la custodia y puesta en valor de los fondos antiguos reunidos por una institución en uno o en diversos centros, como la Biblioteca Marqués de Valdecilla de la Universidad Complutense, o bibliotecas que inician colecciones de fondos antiguos, como las bibliotecas patrimoniales de algunos parlamentos autonómicos españoles (las bibliotecas de las Cortes de Aragón o la del Parlamento Vasco, por ejemplo). Todas ellas están conformando un nuevo tipo de biblioteca que posee características propias.

\section{LA TRASCENDENCIA DE LA VERTIENTE PATRIMONIAL DE LOS FONDOS Y LAS UNIDADES BIBLIOGRÁFICAS ANTIGUAS}

No se pretende profundizar en este aspecto consustancial con las bibliotecas patrimoniales o históricas y con el libro antiguo, pero tampoco se ha de obviar por completo debido a su trascendencia.

Las bibliotecas como conjunto y los fondos antiguos y los libros antiguos como unidad forman parte del patrimonio cultural de los pueblos. Esta afirmación no admite discusión ya que estos fondos y libros representan el conjunto de los conocimientos que han interesado a los antecesores y que han servido de soporte al conocimiento que se posee en la actualidad. Se trata de objetos patrimoniales que deben de ser conservados para su traspaso a las generaciones venideras. Son, junto con el resto de los objetos patrimoniales, la plataforma sobre la que cada pueblo ha consolidado una cultura que le es propia, que lo identifica, que lo pone en relación con otros pueblos en la construcción de culturas nacionales o de la cultura universal. ${ }^{10}$

Si el producto informativo que ofrece una biblioteca histórica o con fondos antiguos no es el mismo que el de otros tipos de bibliotecas, como se ha 
visto, ¿el centro que custodia y difunde esos fondos debería tener las mismas características que el resto de las bibliotecas? Es difícil dar respuesta a esta pregunta. El principal referente bibliotecológico que se podría emplear como elemento más próximo de comparación con la biblioteca con fondos históricos es la biblioteca de investigación -la biblioteca especializada- debido a que ambas ponen el foco de su actividad en la investigación y en la formación, si bien las bibliotecas especializadas hallan su justificación en el empleo de los fondos que custodian: la calidad en su gestión se mide en cantidad de uso. La biblioteca que está conformada por estos bienes patrimoniales, o que custodia en parte bienes patrimoniales, adquiere una obligación con el resto de la sociedad a la que sirve y atiende que no tiene una biblioteca de investigación especializada (mucho menos la biblioteca pública). Por consiguiente, estas bibliotecas, aun asumiendo funciones clásicas como servir de centro de recursos para el aprendizaje ${ }^{11}$ y la investigación, deben servir también como garantía de que los recursos antiguos serán transmitidos en las mejores condiciones posibles a las generaciones venideras. Este principio plantea un dilema: ¿qué posición debe tomar el centro bibliotecario cuando entran en conflicto el uso del recurso y la conservación del mismo? En general la preservación es la misión principal y debe prevalecer, teniendo en cuenta que riesgo no significa daño. La correcta gestión se establece en correcta conservación y, subsidiariamente, en amplia difusión e investigación, ${ }^{12}$ lo que suele dar lugar a normativas específicas. ${ }^{13}$ Esta preocupación por preservar el documento es una de las líneas que establecen la diferencia entre una biblioteca de investigación, en la que lo fundamental es el contenido, y una patrimonial o histórica. Pesa de manera definitiva en este argumento la diferencia evidente en la atención entre documento y contenido.

Esta escueta argumentación deja ver a las claras que la función principal de las bibliotecas con fondos históricos y con libros antiguos es patrimonial, compaginada con la investigación. ¿En qué consiste esa función patrimonial? Ya se ha visto que el aspecto primordial a considerar es la preservación documental. Junto a ésta hay otras consideraciones que también entran de lleno en las funciones de este tipo de bibliotecas.

11 Cf. Juan Miguel Palma Peña, "La socialización del patrimonio bibliográfico y documental de la humanidad desde la perspectiva de los derechos culturales", en Revista General de Información y Documentación, pp. 301-303.

12 Cf. Carlo Federici, "Uso o conservazione? Un falso dilemma", Gazette du livre medieval, 7, 1985, pp. 1-4.

13 Por ejemplo, vid. José Luis Herrera Morillas, "El fondo antiguo de las bibliotecas universitarias de Andalucía, Extremadura y Murcia: colecciones, textos normativos y recursos virtuales", en Boletín de la Asociación Andaluza de Bibliotecarios, 16, 64, 2001, pp. 53-73. 
Carece de sentido conservar para no difundir, de manera que el conocimiento no sea accesible en la actualidad, aun estando al alcance, pero que sí lo sea para las generaciones futuras. Por tanto, como parte de sus funciones la biblioteca patrimonial debe proporcionar el conocimiento de los fondos que posee y hacerlo extensivo a los miembros de la comunidad en la que patrimonialmente se incluye. O lo que es lo mismo, el patrimonio bibliográfico le pertenece a un pueblo que tiene todo el derecho de conocerlo y el deber de protegerlo. La biblioteca debe dedicar a esta labor una buena parte de su actividad como una de sus funciones principales. ${ }^{14}$

Otra función central sería facilitar la investigación sobre sus fondos de manera que no sólo se conozca su existencia sino que, además, se valoren científicamente, se analice su trascendencia y se establezcan las bases de nuevo conocimiento.

Existen más actividades propias de este tipo de bibliotecas entre las que destaca el acrecentamiento del patrimonio mediante la incorporación de nuevos objetos patrimoniales, bien sea porque devienen en patrimoniales por el transcurso natural del tiempo, bien sea mediante la adquisición y recuperación de aquellos objetos patrimoniales que de forma no controlada puedan hallarse. ${ }^{15}$

\section{LA BIBLIOTECA PATRIMONIAL O CON FONDOS ANTIGUOS}

Llegados a este extremo parece necesario valorar si las funciones mencionadas (preservación, difusión y formación, investigación y acrecentamiento) se consideran de la misma manera y con la misma intensidad en otras bibliotecas con fondos modernos.

La preservación de las unidades bibliográficas, fondos y colecciones bibliográficas patrimoniales adquiere una relevancia entre las actividades bibliotecarias que no se encuentra en ningún otro tipo de bibliotecas. Y, por esta causa, en ellas las decisiones de los especialistas de la preservación son vinculantes y afectan a todas las actividades de la biblioteca relacionadas con los libros, que deben ser reglamentadas y supervisadas por dichos especialistas: el modo de almacenamiento y colocación en las estanterías, la forma de consulta, la posibilidad de préstamo, la forma de apertura de intonsos,

14 Vid. Manuel Sánchez Mariana, "Los fondos históricos en las bibliotecas universitarias", en Magán Wals, op. cit., pp. 178-180. 15 Ibid., pp. 173-175. 
el tiempo de permanencia en una exposición, entre otras. ${ }^{16}$ La presencia e importancia de estos profesionales no es tan relevante en otro tipo de bibliotecas, donde su participación no suele estar ni siquiera considerada.

La biblioteca debe dar a conocer los fondos que posee cumpliendo con la obligación de su difusión para su uso y conocimiento. Por lo que respecta a las bibliotecas patrimoniales o históricas, es preciso detenerse en una apreciación de suma importancia: es muy difícil recuperar lo que se pierde o sustrae si se desconoce su existencia y ubicación (porque simplemente se desconoce que se ha poseído); por tanto, la primera medida de preservación es el conocimiento de lo que la biblioteca posee. ${ }^{17}$ El principio general de la difusión se transforma así en una nueva garantía de preservación o, mejor, de recuperación en caso de pérdida. Como se ha dicho anteriormente, cuando se analiza desde una perspectiva social el patrimonio bibliográfico es propiedad del pueblo que lo creó o reunió y el ciudadano tiene el derecho a acceder a él. ${ }^{18}$ ¿Cómo se pueden usar objetos que son delicados por definición y que exigen un uso consciente para que puedan trascender a la generación actual? Son múltiples las posibilidades: los catálogos, la digitalización, la publicación de facsímiles, las exposiciones, las visitas guiadas, etc. ${ }^{19}$ Todos ellos son ejemplos claros de formas de difusión del patrimonio a las que puede acceder cualquier ciudadano sin perjudicar los documentos. Así se consigue cumplir otra función de la biblioteca patrimonial, que es la de servir a los ciudadanos de todas las edades y niveles, sin exclusión, como útil de formación en el conocimiento de su propio patrimonio, de los hombres y las obras que contribuyeron a su cultura y de lo que esa cultura ha supuesto para otros pueblos y para el resto de la humanidad. La mayor parte de los medios empleados para llevar a cabo estas funciones de difusión y formación no se usan en las bibliotecas con fondos modernos o se pueden suplir con fotocopias, que están prohibidas como medio de reproducción cuando se trata de fondos antiguos. De la misma manera, no son frecuentes en las bibliotecas que

16 Por ejemplo: Javier Tacón Clavaín, Cajas de conservación para libros, Documentos de trabajo U.C.M. Biblioteca Histórica, 2010/4; El uso de atriles para la apertura de libros antiguos, Documentos de trabajo U.C.M. Biblioteca Histórica, 2010/5; Procedimiento para la manipulación de códices manuscritos durante la consulta, Documentos de trabajo U.C.M. Biblioteca Histórica, 2011/6; y Procedimiento para la apertura de libros intonsos de fondo antiguo, Documentos de trabajo U.C.M. Biblioteca Histórica, 2011/7, entre otros.

17 De la misma opinión es Idalia García Aguilar, "Entre páginas de libros antiguos: la descripción bibliográfica material en México", en Investigación Bibliotecológica, p. 14.

18 Vid. Palma Peña, op. cit.

19 María Helena Rosales Varo y Luis Villén Rueda, "El acceso y difusión de las colecciones de fondo antiguo: entre la tradición y la modernidad", en XI Jornadas Bibliotecarias de Andalucía, Sevilla, 18, 19, 20 mayo 2000, Jornadas Bibliotecarias de Andalucía (11ª 2000, Alcalá la Real), Sevilla: Asociación Andaluza de Bibliotecarios, Jaén, Diputación Provincial de Jaén, 2000, pp. 287-304. 
poseen fondos modernos la digitalización, la publicación de facsímiles, las exposiciones o las conferencias, por ejemplo.

El investigador que se aproxima a una biblioteca histórica o con fondos antiguos puede tener como objeto de estudio la biblioteca y su contexto, o un conjunto de libros que poseen un procedencia determinada, o un conjunto de encuadernaciones con unas características específicas, o los libros que poseen un determinado exlibris, o las filigranas de un conjunto bibliográfico dado, o un ejemplar con una ilustración especial, u otro representativo de un taller tipográfico o que posee unos paratextos literarios poco conocidos, o los editados por una entidad científica o los desperfectos existentes en ellos. ${ }^{20}$ Ninguna de estas exigencias por parte de los investigadores es común en las bibliotecas que únicamente poseen fondos modernos.

El acrecentamiento patrimonial es otra de las funciones de las bibliotecas patrimoniales o históricas. Pero, ¿cómo se articula? Parece evidente que ha de centrarse, en primer lugar, en recuperar los hijos pródigos que pudieren detectarse; ya se dijo más arriba que si la unidad deja huella en el conjunto también ocurre a la inversa. La principal obligación, por lo que hace referencia a la biblioteca patrimonial o histórica, es adquirir los libros que pertenecieron a las colecciones existentes en la biblioteca y que se perdieron o sustrajeron, incluso con preferencia sobre la adquisición de otros ejemplares que pudieren parecer más interesantes para los fines de la misma. En segundo lugar, se ha de centrar en la adquisición de unidades y colecciones que se adecuen a las políticas de adquisición y acrecentamiento patrimonial del centro en cuestión, que deben ser establecidas de forma previa a cualquier adquisición. También se han de definir los mecanismos por los que los donativos, legados, daciones, etc. se acepten o rechacen. Por descontado, el expurgo como tal no existe en las bibliotecas patrimoniales. Cabe aún diferenciar entre dos conceptos que en este ámbito de estudio se presentan como contrapuestos: fondo y colección. De nuevo, las diferencias entre ambos tipos de bibliotecas se aprecian de forma evidente. De manera general, un fondo es un conjunto documental que llega hasta el presente y se caracteriza por poseer un origen común que lo dota de sentido, por lo que debe mantenerse reunido; una colección es un conjunto documental creado con unos fines específicos que evoluciona constantemente para cumplirlos, incorporando nuevos documentos adecuados y útiles y relegando otros obsoletos fuera de uso. Las bibliotecas patrimoniales suelen poseer fondos adquiridos o heredados de la propia institución, mientras que las bibliotecas con fondos modernos crean colecciones.

20 David Asencio Padilla, Miguel Ángel Sánchez Herrador e Inmaculada Carrasco Pleguezuelo, "El deterioro del libro antiguo como fuente de información histórica", en Revista General de Información y Documentación, 20, 1, 2010, pp. 281-296. 
El análisis de sus funciones principales revela que las bibliotecas históricas o patrimoniales poseen peculiaridades que las individualizan con respecto a otras tipologías bibliotecarias. Cabe aun analizar la manera en la que inciden estos fondos y sus características en las actividades cotidianas de la biblioteca o, lo que es lo mismo, determinar si esas cuatro funciones afectan al modo de proceder en las bibliotecas patrimoniales o con fondos antiguos. Para dar respuesta a esta incógnita se han de analizar las consecuencias biblioteconómicas que produce la relación entre los libros y las colecciones antiguas y los investigadores y el público en general, y cómo la biblioteca se adapta a esa relación adoptando, muchas veces de forma intuitiva, soluciones eficientes y eficaces para adecuarse a los fines que le son propios y que previamente se han enunciado. En algunos casos, frente a los problemas que se plantean, se actúa mediante el refuerzo de actitudes y actuaciones presentes en otro tipo de bibliotecas; en otros, se ofrecen soluciones novedosas en el mundo de la biblioteconomía. Para ello, de forma somera, se trata la selección, el tratamiento y la difusión documental.

Hablar de selección, cuando se trata de fondos antiguos, implica el conocimiento del mercado anticuario del libro, que sigue vías muy diferentes de las de los libros modernos, así como atender qué es lo que se selecciona: por supuesto el ejemplar de una edición, que es diferente al resto de los que se conservan y que tiene sentido en la biblioteca por su encuadernación, su autoría, sus preliminares, sus poseedores, sus ilustraciones, por el lugar en el que se imprimió, etc. Entonces, aunque en todas las bibliotecas se seleccionan libros, éstos no tienen por qué poseer las mismas características. Los libros antiguos pueden estar o no en el mercado; cuando lo están pueden sufrir variaciones de precio notables en función de las características del ejemplar, características que además le confieren interés para la biblioteca o que resultan absolutamente indiferentes para la misma. De la misma manera se puede seleccionar un fondo con base en la procedencia; esto es, quién, cuándo, cómo y por qué se crea ese fondo o biblioteca, lo que no invalida el interés por las unidades que lo componen, todas o parte de ellas.

El tratamiento seguido en unas y otras bibliotecas difiere desde lo más elemental, como la forma de indicar el número de registro en el libro, de sellarlo o de poner el tejuelo, hasta los aspectos más técnicos como la forma de establecer las características del ejemplar en los registros bibliográficos. Es preciso tener presente que no se puede catalogar un libro antiguo sin identificarlo previamente, para lo que es preciso utilizar determinadas herramientas. Se trata de las características propias de la catalogación de este tipo de libros que no afectan de la misma manera a los libros modernos. Pero hay muchas más, como los cuidados en el almacenamiento de los fondos que son 
mucho mayores para los volúmenes de forma independiente con cajas que eviten las afecciones externas, la adecuación de los procesos de descripción a las características del ejemplar sin romper el principio de identidad en la descripción para ejemplares de la misma edición, la indicación de referencias bibliográficas sobre la edición, la extracción de encabezamientos por el nombre del impresor existiendo el de editor, etcétera.

Además, la investigación sobre estos fondos precisa de la presencia de elementos especiales como las hojas de luz para el estudio de filigranas, que los libros deban ser consultados sobre atriles especiales que eviten el forzado de las encuadernaciones y el uso preceptivo de guantes y lápiz, por ejemplo; así como seguir acciones específicas como la supervisión de los bibliotecarios sobre las actividades de investigación, que frecuentemente requieren reproducciones de alta calidad, o la revisión estricta de los objetos que se introducen y sacan de la biblioteca, entre otras.

Se ha tratado ya de las especificidades que poseen las bibliotecas patrimoniales en cuanto a la difusión. La celebración de exposiciones, la elaboración de catálogos, la edición de facsímiles, la celebración de reuniones científicas y de conferencias, las visitas guiadas, la adecuación de todas estas actividades a personas de muy diversa preparación y edad son actividades, en su mayor parte, exclusivas de las bibliotecas patrimoniales.

\section{A MODO DE CONCLUSIÓN}

Por tanto, al hablar de bibliotecas patrimoniales o bibliotecas históricas nos referimos a un tipo de centros de información, con génesis diversas, que recientemente está adquiriendo trascendencia. Poseen características específicas que derivan, en primer lugar, de las peculiaridades de los documentos que custodian, que pueden ser objeto de estudio como unidades o como fondo o colección; en segundo lugar, del componente patrimonial que poseen. Son precisamente estos pormenores los que conducen a que las funciones de estas bibliotecas se centren en cuatro apartados de forma destacada: preservación, difusión y formación, investigación y acrecentamiento, en ese orden. También son estos mismos factores los que afectan a muchas de las actuaciones técnicas que se siguen en estas bibliotecas.

Todo ello conduce a la deducción de que las bibliotecas patrimoniales deben ser entendidas como un tipo de bibliotecas que tienen características propias más allá de la perspectiva de la especialización; que requieren medios y espacios distintos a los de otro tipo de bibliotecas así como personal técnico y de gestión con conocimientos específicos, por lo general diferentes 
de los que se precisan para otro tipo de bibliotecas. ${ }^{21}$ Finalmente, habrá de considerarse la pronta inclusión de una especialidad en este tipo de centros como una de las vertientes de la formación de los bibliotecarios. ${ }^{22}$

\section{BibliografíA}

Asencio Padilla, David; Miguel Ángel Sánchez Herrador e Inmaculada Carrasco Pleguezuelo, "El deterioro del libro antiguo como fuente de información histórica", en Revista General de Información y Documentación, 20, 1, 2010, pp. 281-296.

Báez, Fernando, Historia universal de la destrucción de libros, Barcelona: Destino, 2004.

_- Nueva historia universal de la destrucción de libros, Barcelona: Seix Barral, 2011.

Carrión Gútiez, Manuel, "Tres lecciones sobre la Biblioteca Nacional de España”, en Ramón Rodríguez Álvarez y Moisés Llordén Miñambres, El libro antiguo en las bibliotecas españolas, Oviedo: Universidad de Oviedo, Servicio de Publicaciones, 1998, pp. 9-38.

Federici, Carlo, "Uso o conservazione? Un falso dilemma", Gazette du livre medieval, 7, 1985, pp. 1-4.

García Aguilar, Idalia, "Entre páginas de libros antiguos: la descripción bibliográfica material en México", en Investigación bibliotecológica, 22, 45, 2008, pp. 13-40.

Gaskell, Philip, Nueva introducción a la bibliografía material, trad. de la ed. de Oxford University Press, Gijón: Trea, 1998 [1972].

Herrera Morillas, José Luis, "El fondo antiguo de las bibliotecas universitarias de Andalucía, Extremadura y Murcia: colecciones, textos normativos y recursos virtuales", en Boletín de la Asociación Andaluza de Bibliotecarios, 16, 64, 2001, pp. 53-73.

Magán Wals, José Antonio, Temas de biblioteconomía universitaria y general, Madrid: Universidad Complutense, 2002.

Martín Abad, Julián, "El bibliotecario de manuscritos e impresos antiguos en España: misión y circunstancias", en Bibliotecas: puentes hacia universos culturales más amplios, Reunión Nacional de Bibliotecarios (41ㄹ 2009, Buenos Aires), Buenos Aires: Asociación de Bibliotecarios Graduados de la República Argentina, 2009. Disponible en: http://www.bne.es/es/Micrositios/Guias/JMAbad/ Bibliografia/2009-2012/ (Fecha de consulta: abril de 2013).

21 Vid. Julián Martín Abad, "El bibliotecario de manuscritos e impresos antiguos en España: misión y circunstancias", en Bibliotecas: puentes hacia universos culturales más amplios, Reunión Nacional de Bibliotecarios (41‥ 2009. Buenos Aires), Buenos Aires: Asociación de Bibliotecarios Graduados de la República Argentina, 2009.

22 Vid. Raphaële Mouren (ed.), Ambassadors of the book: competences and training for heritage librarians, The Hague: IFLA, De Gruyter Saur, 2012 
Martín Abad, Julián, La valoración del libro: el punto de vista del bibliotecario de fondo antiguo, Documentos de trabajo U.C.M. Biblioteca Histórica, 8, 2004, pp. 1-25. Disponible en: http://eprints.ucm. es/5698/1/2004-8.pdf (Fecha de consulta: abril de 2013).

McKerrow, Ronald B., Introducción a la bibliografía material, Madrid: Arco/Libros, 1998.

Moll, Jaime, "Problemas bibliográficos del libro del Siglo de Oro", en Boletín de la Real Academia Española, 59, 216, 1979, pp. 49-107.

Mouren, Raphaële (ed.), Ambassadors of the book: competentes and trining for Heritage librarians, The Hague: IFLA, De Gruyter Saur, 2012.

Palma Peña, Juan Miguel, "La socialización del patrimonio bibliográfico y documental de la humanidad desde la perspectiva de los derechos culturales", en Revista General de Información y Documentación, 21, 2011, pp. 291-312.

Pedraza Gracia, Manuel José, "La valoración de fondos antiguos en bibliotecas", en Ibersid: revista de sistemas de información y comunicación, 2, 2008, pp. 263-272.

Rosales Varo, María Helena y Luis Villén Rueda, "El acceso y difusión de las colecciones de fondo antiguo: entre la tradición y la modernidad", en XI Jornadas Bibliotecarias de Andalucía, Sevilla, 18, 19, 20 mayo 2000, Jornadas Bibliotecarias de Andalucía (11"a 2000, Alcalá la Real), Sevilla: Asociación Andaluza de Bibliotecarios, Jaén, Diputación Provincial de Jaén, 2000, pp. 287-304.

Sánchez Mariana, Manuel, "Los fondos históricos en las bibliotecas universitarias”, en José Antonio Magán Wals (ed.), Temas de biblioteconomía universitaria y general, Madrid: Universidad Complutense, 2002, pp. 166-190.

Tacón Clavaín, Javier, Cajas de conservación para libros, Documentos de trabajo U.C.M. Biblioteca Histórica, 2010/4. Disponible en: http://pendientedemigracion.ucm.es/BUCM/foa/37924.php (Fecha de consulta: abril de 2013).

_- El uso de atriles para la apertura de libros antiguos, Documentos de trabajo U.C.M. Biblioteca Histórica, 2010/5. Disponible en: http: //pendientedemigracion.ucm.es/BUCM/foa/37928.php (Fecha de consulta: abril de 2013).

— - Procedimiento para la manipulación de códices manuscritos durante la consulta, Documentos de trabajo U.C.M. Biblioteca Histórica, 2011/6. Disponible en: http://pendientedemigracion.ucm. es/BUCM/foa/doc16877.pdf (Fecha de consulta: abril de 2013).

_- Procedimiento para la apertura de libros intonsos de fondo antiguo, Documentos de trabajo U.C.M. Biblioteca Histórica, 2011/7. Disponible en: http://pendientedemigracion.ucm.es/BUCM/foa/ doc16876.pdf (Fecha de consulta: abril de 2013).

Tugores, Francesca y Rosa Planas, Introducción al patrimonio cultural, Gijón: Trea, 2006. 